\title{
Um modelo fracionário logístico com medidas de controle para casos acumulados de COVID-19: cenários no Brasil
}

\author{
Michele Martins Lopes ${ }^{1}$ \\ IMECC/Unicamp, Campinas, SP \\ Francielle Santo Pedro ${ }^{2}$ \\ DMD/Unifesp, Osasco, SP \\ Daniel Sánchez Ibáñez ${ }^{3}$ \\ Centro Docencia Cs. Básicas para Ingeniería /UACh, Valdivia, Chile. \\ Vinícius Francisco Wasques ${ }^{4}$ \\ DM/Unesp, Rio Claro, SP \\ Estevão Esmi ${ }^{5}$ \\ IMECC/Unicamp, Campinas, SP \\ Laécio Carvalho de Barros ${ }^{6}$ \\ IMECC/Unicamp, Campinas, SP
}

Resumo. A curva de casos acumulados de indivíduos infectados por COVID-19 apresenta crescimento similar ao logístico, no período referente à cada "onda" epidêmica, como é chamado cada pico de casos ativos. Considerando que em cenários de pandemia é comum buscar medidas de controle com base em experiências prévias, neste trabalho buscamos modelar a curva de casos acumulados através de um modelo logístico com retirada, para representar o controle, baseado em equações diferenciais fracionárias para incluir o efeito de memória. Com isso, apresentamos projeções dos cenários com e sem controle, buscando prever o número de infectados por coronavírus no Brasil.

Palavras-chave. Coronavírus, Equações Diferencias Fracionárias, Isolamento Social.

\section{Introdução}

Em dezembro de 2019, um surto de pneumonia ligado a um mercado de frutos do mar, que comercializava animais vivos em Wuhan na China, chamou a atenção do mundo. Em fevereiro de 2020, a Organização Mundial da Saúde (World Health Organization, em inglês) designou essa doença por COVID-19. O acrônimo vem de "corona virus deceased 2019", mas anteriormente era chamada de 2019-nCov [5, 12].

Os coronavírus são importantes patógenos humanos e animais. Eles são conhecidos por causar infecção respiratória [12]. Desde o início do milênio já tivemos três novos coronavírus. Em 2002, surgiu a SARS que causa a síndrome respiratória aguda grave, depois em 2012 surgiu a síndrome respiratória do Oriente Médio, chamada MERS e, por último, a COVID-19. A fonte primária dos coronavírus são os morcegos. A grosso modo, os coronavírus "saltam" de uma espécie animal, o qual é um parasita habitual para uma espécie humana [5]. No caso da SARS o pangolim foi o

\footnotetext{
1 mi_martins22@hotmail.com

2 fsimoes@unifesp.br

3 danielsanch@gmail.com

${ }^{4}$ viniwasques@hotmail.com

5 eelaureano@gmail.com

${ }^{6}$ laeciocb@ime.unicamp.br
} 
intermediário com os humanos, e no caso da MERS foi o dromedário. Ainda é desconhecido se a COVID-19 é transmitida diretamente de morcegos ou através de hospedeiro intermediário [9].

Segundo o sequenciamento de genoma completo e a análise filogênica, o coronavírus que causa a COVID-19 é um betacoronavírus. A estrutura da região do gene de ligação ao receptor é muito semelhante à do coronavírus da SARS [17].

A compreensão do risco de transmissão ainda está incompleta. No entanto, o início do surto em Wuhan teve uma associação com o mercado de peixes e à medida que o surto progredia, a disseminação de pessoa para pessoa se tornou o principal modo de transmissão [14].

Acredita-se que a disseminação de pessoa para pessoa da COVID-19 ocorra principalmente por gotículas respiratórias, semelhante à da influenza. $\mathrm{O}$ vírus é liberado nas secreções respiratórias quando uma pessoa com infecção tosse, espirra ou fala e, através da transmissão de gotículas, infecta outra pessoa ao entrar em contato direto com as membranas mucosas. As gotículas normalmente não viajam mais de dois metros e, aparentemente, não permanecem no ar [11].

Os dados indicam que a transmissão é mais provável no estágio inicial da infecção, pois os níveis de ácido ribonucleico (RNA) viral parecem estar mais altos logo após o início dos sintomas da doença [18]. Segundo [8], acredita-se que o período de incubação da COVID-19 esteja em torno de 14 dias após a exposição, sendo que a maioria dos casos ocorreram aproximadamente de quatro a cinco dias após a exposição. Evidências preliminares sugerem que alguns anticorpos induzidos naqueles que foram infectados são protetores. Porém, ainda não se sabe se todos os pacientes infectados apresentam uma resposta imune protetora e quanto tempo durará algum efeito protetor [1].

Complicações graves da doença podem ocorrer em indivíduos saudáveis de qualquer idade, mas ocorre predominantemente em adultos com idade avançada ou comorbidades médicas subjacentes $[5,16]$. Aparentemente, a pneumonia é a manifestação mais grave da infeç̧ão. Ela é caracterizada principalmente por febre, tosse, dispnéia e infiltrados bilaterais na imagem torácica $[7,8]$.

Para reduzir o risco de transmissão na comunidade, recomenda-se o distanciamento social. Além disso, os indivíduos devem lavar as mãos diligentemente, praticar a higiene respiratória (por exemplo, cobrir a tosse), evitar multidões e contato próximo com indivíduos doentes [13].

Em situações de pandemia é comum considerar que no decorrer do tempo as pessoas passem a tomar medidas de prevenção para controle da doença e, para isso, considerarem experiências prévias. Em vista disso, nesse trabalho analisamos os cenários da COVID-19 considerando medidas de controle, como em [6], e memória.

Em [10] podemos ver que, dentre várias formas de se introduzir memória em um modelo de equações diferenciais ordinárias, uma forma é torná-las equações diferenciais fracionárias, utilizando a derivada fracionária de Caputo. Em [2] os autores trabalham com outro modelo fracionário para estudar a COVID-19, obtendo bons resultados.

\section{Modelagem Matemática}

Essa seção apresenta o modelo usado neste trabalho para descrever a evolução da pandemia causada pelo coronavírus (COVID-19) até o presente momento. Desse modo, apenas será analisada a evolução dos indivíduos confirmadamente infectados pela doença, através da curva de casos acumulados. Para descrever a velocidade em que a contaminação se propaga é utilizado um crescimento logístico [4] com memória, através da equação diferencial fracionária logística, com derivada de Caputo. Em [10] vemos que de fato uma equação diferencial fracionária com derivada de Caputo apresenta memória. O modelo utilizado ainda apresenta retirada de indivíduos infectados como medida de controle [6]. Tal retirada se dá pelo isolamento de infectados ou de toda a população, que está sendo adotada em diversos países do mundo. 
O modelo proposto é descrito pelo seguinte Problema de Valor Inicial (PVI)

$$
\left\{\begin{array}{l}
D_{t}^{\alpha} I=r^{\alpha} I\left(1-\frac{I}{K}\right)-p^{\alpha} I, \\
I(0)=I_{0}
\end{array}\right.
$$

em que $I(t)$ é o número de casos confirmados de infecção pelo coronavírus no tempo $t$, dado em dias, $r$ é a taxa de contágio com mobilidade livre de pessoas, $K$ é a capacidade de suporte do meio, $p$ é a parcela de indivíduos removidos do meio, por conta do isolamento social ou outras medidas de controle, e $\alpha \in(0,1)$ é a ordem fracionária da derivada de Caputo.

\subsection{Análise do modelo}

Inicialmente, (1) pode ser entendido como uma extensão do modelo clássico $(\alpha=1)$ [4]. Neste caso, um equilíbrio assintoticamente estável seria dado por $\bar{I}=\left(\frac{r-p}{r}\right) K$ e, quando $p=0$, temos $\bar{I}=K$. Ou seja, $p$ pode ser visto como uma medida de controle da epidemia. No modelo fracionário (1) os parâmetros $r$ e $p$ devem ser elevados a $\alpha$ devido ao estudo de análise dimensional [3].

Uma manipulação algébrica nos permite reescrever (1) na forma

$$
D_{t}^{\alpha} I=\left(r^{\alpha}-p^{\alpha}\right) I\left(1-\frac{I}{\left(\frac{r^{\alpha}-p^{\alpha}}{r^{\alpha}}\right) K}\right),
$$

donde temos uma nova capacidade de suporte: $\bar{K}=\left(\frac{r^{\alpha}-p^{\alpha}}{r^{\alpha}}\right) K$.

De acordo com [2], para $\alpha \in(0,1)$,

$$
D_{t}^{\alpha} I=\frac{t^{1-\alpha}}{\Gamma(2-\alpha)} E\left[I^{\prime}(t W)\right]
$$

onde $W$ é uma variável aleatória com distribuição beta de parâmetros 1 e $1-\alpha$, isto é, $W \sim B(1,1-\alpha)$. Assim, $D_{t}^{\alpha} I$ está relacionada com a esperança matemática de $I^{\prime}(s), 0 \leq s \leq t$. No caso aqui estudado, $I(s)$ representa casos acumulados, de maneira que $I^{\prime}(s) \geq 0$. Logo, $D_{t}^{\alpha} I \geq 0$. Esse fato implica $r^{\alpha} \geq p^{\alpha}(r \geq p)$, ou seja, a taxa de contágio $(r)$ é sempre superior à taxa de removidos do convívio social $(p)$.

Para o caso aqui estudado, como $I^{\prime}(t) \geq 0$, o equilíbrio é caracterizado por um valor constante em que $I(t)$ se aproxima dele assintoticamente. De acordo com $(2)$, esse valor é $\bar{K}=\left(\frac{r^{\alpha}-p^{\alpha}}{r^{\alpha}}\right) K$. Isto é, quando $t \rightarrow \infty$,

$$
I(t) \rightarrow\left(\frac{r^{\alpha}-p^{\alpha}}{r^{\alpha}}\right) K
$$

Assim, se $p=0, I(t) \rightarrow K$. Por outro lado, se $p \neq 0$, temos $\left(\frac{r^{\alpha}-p^{\alpha}}{r^{\alpha}}\right) K<K$. Isto é, a nova capacidade de suporte, no cenário com controle, é menor que aquela do cenário sem controle $(p=0)$. Mais ainda, a curva $I(t)$ tende a estabilizar em um valor $(\bar{K})$ tanto menor quanto maior for o valor de $p$.

\section{Aplicação Numérica}

Nessa seção, vamos usar o modelo dado em (2) para realizar uma simulação através de dados reais de casos acumulados no Brasil, obtidos em [15]. Os dados foram coletados desde o primeiro caso, em 25 de fevereiro de 2020, até a data da atualização do trabalho, em 10 de março de 2021. 
É importante notar que através da curva de casos ativos da doença no Brasil, assim como em outros países, podemos observar a chamada "segunda onda" se iniciando. No Brasil isso ocorreu em torno da segunda semana de novembro de 2020. Em contrapartida, analisando os casos acumulados (que, devemos lembrar, apenas aumenta), no período referente à cada "onda", podemos concluir que inicialmente o número de casos cresce exponencialmente. Então, a partir do pico da "onda" (onde casos ativos começam a decrescer), o número de casos acumulados passa a crescer mais lentamente, donde temos um ponto de inflexão. Quando o número de casos ativos para de crescer (fim da "primeira onda", antes de iniciar a segunda) é quando o número de casos acumulados tende a estabilizar. Assim, vemos um crescimento similar ao logístico na curva de casos acumulados no período referente à cada "onda" de casos ativos.

Com isso, dividimos os dados reais em duas partes: primeira parte referente ao período em que ocorre a "primeira onda" e a segunda parte referente ao período da "segunda onda", que ainda não se completou. Então, utilizamos o nosso modelo em cada parte dos dados separadamente. Vale ressaltar que se não houvesse uma "segunda onda", como foi o caso da China, por exemplo, então o modelo poderia captar a propagação do coronavírus em todo o período da pandemia, não havendo a necessidade de separar os dados em duas partes.

Em cada parte, foi realizado um ajuste de dados através de quadrados mínimos. Com isso, encontramos os quatro parâmetros utilizados no modelo: a ordem da derivada $(\alpha)$, a taxa de contágio $(r)$, a constante $K$ que compõe a nova capacidade de suporte do modelo $\bar{K}=\left(\frac{r^{\alpha}-p^{\alpha}}{r^{\alpha}}\right) K$ e, por fim, o parâmetro $p$ de remoção de indivíduos, caracterizando o controle adotado no respectivo período. O parâmetro $r$ encontrado no período da "primeira onda" foi fixado para o da "segunda onda". Para encontrar a solução de (2) utilizamos o método numérico de Adams-Bashforth-Moulton preditor corretor fracionário. Utilizamos o valor inicial $I(0)$ como sendo o primeiro valor observado nos dados reais de casos ativos por COVID-19, nas respectivas "ondas".

Vimos que $p=0$ representa nenhuma medida de controle. Assim, é possível fazer uma projeção do cenário da pandemia caso nenhuma medida de controle houvesse sido adotada. Vale lembrar que, neste cenário sem controle, a capacidade de suporte da curva é $K$.

\subsection{Cenário da "primeira onda"}

Analisamos, primeiramente, o cenário da "primeira onda". Os valores obtidos para os parâmetros foram, aproximadamente, $\alpha=0,277, r=0,276, K=12,068 \cdot 10^{6}$ e $p=0,001$.

Como $\alpha$ se aproxima mais de 0 do que de 1 (caso clássico, sem memória) podemos concluir que neste período houve bastante efeito de memória. Por outro lado, o parâmetro $p=0,001$ indica pouco controle, relativamente. Ainda, é possível analisar as capacidades de suporte do cenário com controle e sem controle, que são, respectivamente,

$$
\bar{K}=9,253 \cdot 10^{6} \quad \text { e } \quad K=12,068 \cdot 10^{6} .
$$

Na Figura 1, a curva azul representa os dados reais, a vermelha representa a projeção do cenário sem isolamento (que teria ocorrido se $p=0$ ) e a verde representa o cenário com isolamento. Este último, que de fato ocorreu, foi projetado para os próximos 420 dias (14 meses - abrangendo todo o ano de 2021), cenário que teria ocorrido caso não houvesse "segunda onda".

Podemos ver que o modelo fracionário apresenta bom ajuste aos dados. Apesar do pouco controle captado pelo modelo, na simulação do cenário com isolamento ocorreram aproximadamente 3 milhões de casos confirmados a menos do que ocorreriam no cenário sem isolamento. Neste último, vemos que o número de casos confirmados tende a crescer de forma mais rápida.

Devido ao baixo valor de $\alpha$ as curvas crescem lentamente, não sendo possível observar o limite das curvas. No entanto, aumentando demasiadamente o número de meses de projeção é possível observar que de fato convergem para os valores de capacidade de suporte acima mencionados. 


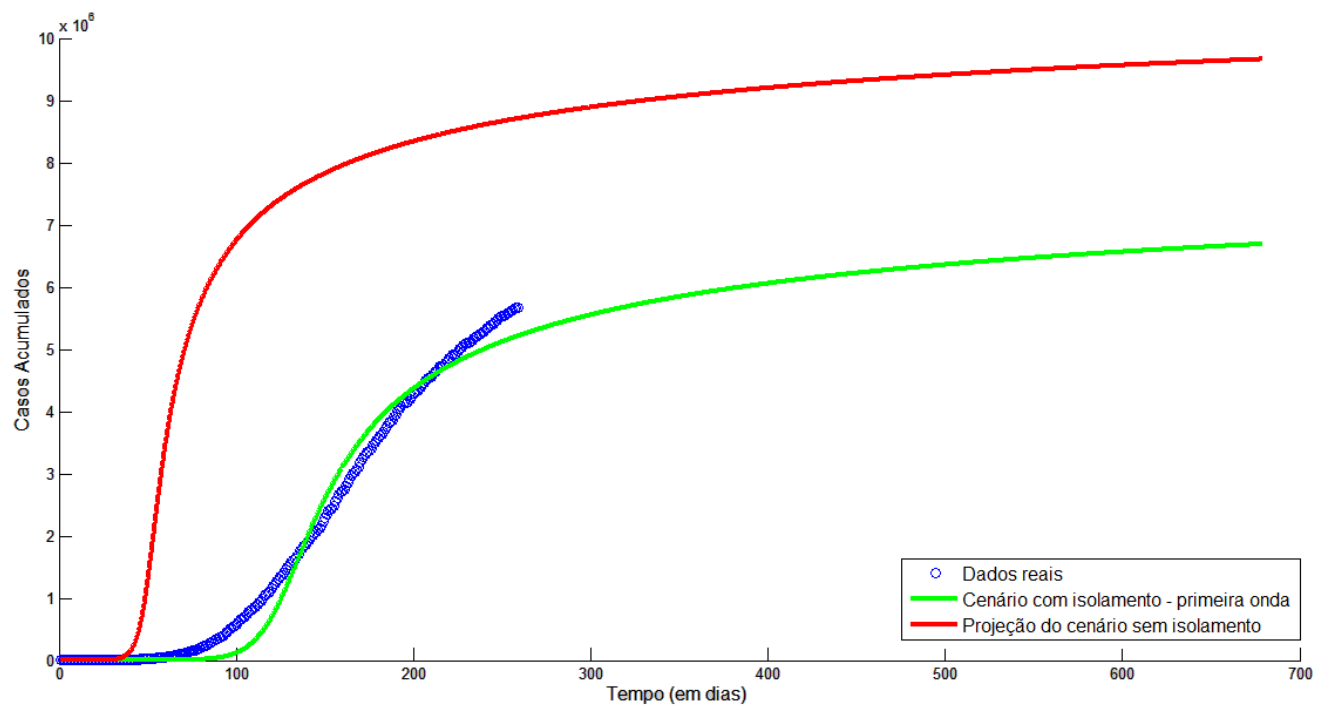

Figura 1: Soluções do modelo (2) nos cenários com e sem isolamento na "primeira onda", em relação aos dados reais. Para esse cenário, $\alpha=0,277, r=0,276, K=12,068 \cdot 10^{6}$ e $p=0,001$.

\subsection{Cenário com a "segunda onda"}

Agora vamos analisar o cenário da "segunda onda", que representa o contexto atual. Os valores obtidos para os parâmetros foram, aproximadamente, $\alpha=0,999, r=0,276, K=1,674 \cdot 10^{9} \mathrm{e}$ $p=0,269$.

Como $\alpha \sim 1$, se assemelhando ao caso clássico, concluímos que durante a "segunda onda" o efeito de memória não é mais utilizado como na primeira onda, praticamente deixando de existir. Por outro lado, o parâmetro $p$ indica que há mais controle nesse período do que no referente à "primeira onda". A capacidade de suporte deste novo cenário, com controle, foi

$$
\bar{K}=42,083 \cdot 10^{6} \text {. }
$$

Na Figura 2, a curva azul representa os dados reais, a verde representa o cenário com isolamento da "primeira onda" e a roxa o cenário com isolamento da "segunda onda". Para este caso também foi feita uma projeção abrangendo todo o ano de 2021, isto é, para os próximos 270 dias (9 meses). A linha vertical preta tracejada representa a separação dos dados entre as duas "ondas". É possível ver que na curva de casos acumulados há um ponto de inflexão quando a nova "onda" se inicia.

Podemos ver, novamente, que o modelo fracionário apresenta ótimo ajuste aos dados. É claro que o surgimento da "segunda onda" proporciona maior número de casos confirmados e, para esse contexto, estimamos que o número total de casos confirmados chegará a 42 milhões antes de a pandemia encerrar completamente, dentro de aproximadamente 2 anos.

\section{Conclusões}

O modelo logístico que inclui efeito de memória e medida de controle, aqui apresentado, mostra bons resultados para representar a curva de casos acumulados no período referente à cada "onda". Ele apresenta ótimo ajuste aos dados, proporcionando a capacidade de estimar um cenário futuro, bem como projetar um cenário fictício caso não houvesse controle. As simulações, que analisam a situação do Brasil, comprovam a teoria de que quanto maior o controle, como isolamento social, 


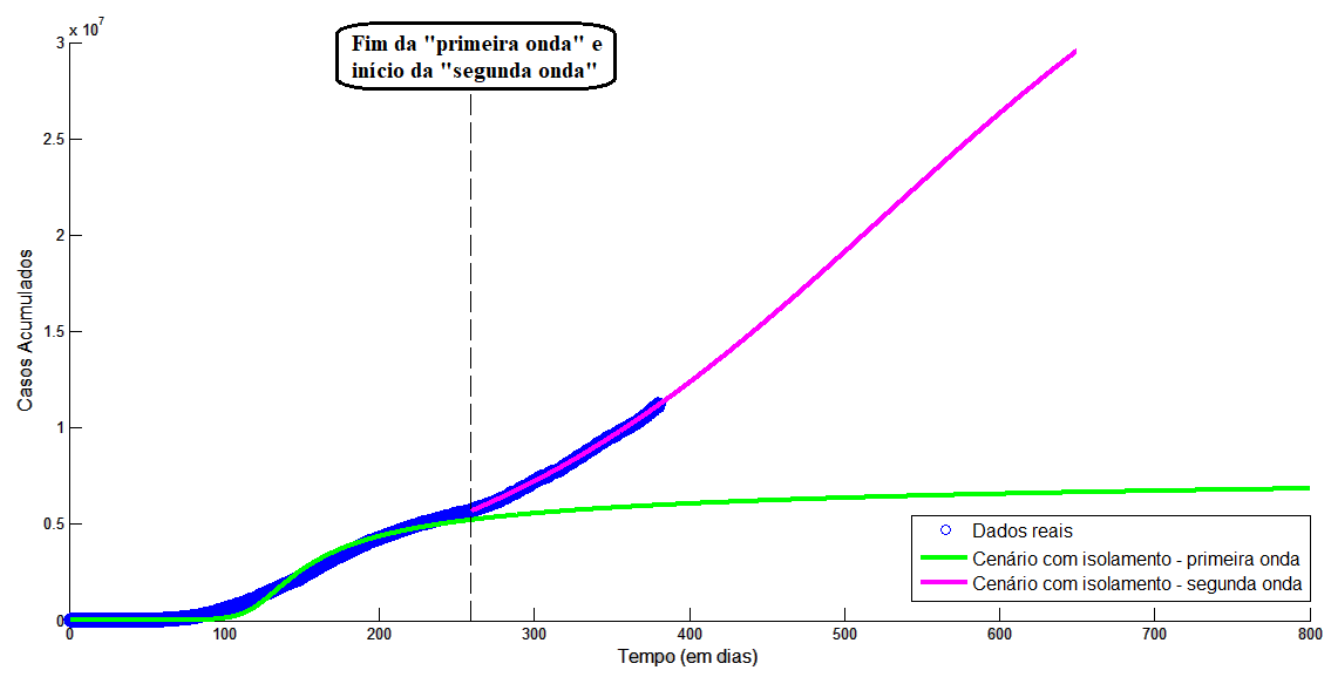

Figura 2: Soluções do modelo (2) para o cenário com isolamento na primeira e na segunda "onda", em relação aos dados reais. Para esse cenário, $\alpha=0,999, r=0,276, K=1,674 \cdot 10^{9}$ e $p=0,269$.

melhor o cenário diante da pandemia que enfrentamos, diminuindo o número de casos da doença. Além do mais, se não houver novas "ondas", nosso trabalho permite estimar a quantidade de casos acumulados que chegaremos a atingir quando a pandemia se encerrar completamente: aproximadamente 42 milhões de infectados em aproximadamente 2 anos. No entanto, se não forem mantidas, reforçadas ou melhoradas as medidas de controle atuais, a tendência é surgir novas "ondas" e, com isso, novos pontos de inflexão que dão origem à várias outras curvas logísticas "conectadas", como vemos na Figura 2.

Na dinâmica do nosso modelo é bem retratada a variação da capacidade de suporte de infectados para diferentes valores de $p$, que representa a presença de medidas de controle e é determinante para desacelerar o crescimento da curva de infectados conforme maior o isolamento. Isso fica claro em nosso trabalho, no qual mostramos que o aumento de $p$ implica em diminuição na capacidade de suporte, isto é, quanto maior a medida de controle, menor será o número de casos confirmados com COVID-19.

\section{Agradecimentos}

O presente trabalho foi realizado com apoio da Coordenação de Aperfeiçoamento de Pessoal de Nível Superior - Brasil (CAPES) - Código de Financiamento 001, e com apoio do CNPq sob o registro número 306546/2017-5.

\section{Referências}

[1] Bao, L., Deng, W., Gao, H., Xiao, C., et al. Reinfection could not occur in SARS-CoV-2 infected rhesus macaques. BioRxiv, 2020.

[2] Barros, L. C., Lopes, M. M., Santo Pedro, F., Esmi, E., dos Santos, J. P. C. and Sánchez, D. E. The memory effect on fractional calculus: an application in the spread of COVID-19. Comp. Appl. Math., 40(3): 1-21, 2021. DOI: 10.1007/s40314-021-01456-z. 
[3] Diethelm, K. A. fractional calculus based model for the simulation of an outbreak of dengue fever. Nonlinear Dynamics 71(4): 613-619, 2013.

[4] Edelstein-Keshet, L. Mathematical models in biology. Society for Industrial and Applied Mathematics, 2005.

[5] Fundação Oswaldo Cruz. Covid-19: que vírus é esse? https://portal.fiocruz.br/noticia/covid19-que-virus-e-esse (Acessado em março de 2020).

[6] Góis, A. N., Laureano, E. E., Santos, D. D. S., Sánchez, D. E., et al. Lockdown as an Intervention Measure to Mitigate the Spread of COVID-19: a modeling study. SBMT, 53, 2020.

[7] Huang, C., Wang, Y., Li, X., Ren, L., et al. Clinical features of patients infected with 2019 novel coronavirus in Wuhan, China. The Lancet, 395.10223:497-506, 2020. DOI: 10.1016/S01406736(20)30183-5.

[8] Li, Q., Guan, X., Wu, P., Wang, X., et al. Early transmission dynamics in Wuhan, China, of novel coronavirus-infected pneumonia. NEJM, 382: 1199-1207, 2020. DOI: 10.1056/NEJMoa2001316.

[9] Perlman, S. Another decade, another coronavirus. NEJM, 760-762, 2020. DOI: 10.1056/NEJMe2001126.

[10] Saeedian, M., Khalighi, M., Azimi-Tafreshi, N., Jafari, G. R. and Ausloos, M. Memory effects on epidemic evolution: The susceptible-infected-recovered epidemic model. Physical Review, 95: 0224091-0224099, 2017. DOI: 10.1103/PhysRevE.95.022409.

[11] Van Doremalen, N., Bushmaker, T., Morris, D. H., Holbrook, M., et al. Aerosol and surface stability of SARS-CoV-2 as compared with SARS-CoV-1. NEJM, 2020. DOI: 10.1056/NEJMc2004973.

[12] World Health Organization. Director-General's remarks at the media briefing on 2019-nCoV on 11 February 2020. https://www.who.int/dg/speeches/detail/who-director-general-s-remarksat-the-media-briefing-on-2019-ncov-on-11-february-2020 (Acessado em março de 2020).

[13] World Health Organization. Novel Coronavirus (2019-nCoV) technical guidance. https://www.who.int/emergencies/diseases/novel-coronavirus-2019/technical-guidance (Acessado em março de 2020).

[14] World Health Organization. Novel coronavirus situation report -2. January 22, 2020. https://www.who.int/docs/default-source/coronaviruse/situation-reports/20200122-sitrep-22019-ncov.pdf (Acessado em março de 2020).

[15] Worldometers. Covid-19 coronavirus pandemic, https://www.worldometers.info/coronavirus/ country/brazil/. (Acessado em março de 2021)

[16] Zhou, F., Yu, T., Du, R., Fan, G., et al. Clinical course and risk factors for mortality of adult inpatients with COVID-19 in Wuhan, China: a retrospective cohort study. The Lancet, 395:1054-1062, 2020. DOI: 10.1016/S0140-6736(20)30566-3.

[17] Zhou, P., Yang, X. L., Wang, X. G., Hu, B., et al. A pneumonia outbreak associated with a new coronavirus of probable bat origin. Nature, 1-4, 2020. DOI: 10.1038/s41586-020-2012-7.

[18] Zou, L., Ruan, F., Huang, M., Liang, L., et al. SARS-CoV-2 viral load in upper respiratory specimens of infected patients. NEJM, 382.12: 1177-1179, 2020. DOI: 10.1056/NEJMc2001737. 